\title{
A pilot randomized controlled trial of omega-3 fatty acid supplementation for the treatment of anxiety in adolescents with anorexia nervosa
}

\author{
Brittny E. Manos BA ${ }^{1}$ | Terrill D. Bravender MD, MPH ${ }^{2}$ | Tondi M. Harrison PhD ${ }^{1,3}$ | \\ Hannah L. H. Lange $\mathrm{MPH}^{1}$ | Casey B. Cottrill MD, $\mathrm{MPH}^{3,4}$ | \\ Mahmoud Abdel-Rasoul MS, MPH ${ }^{3}$ | Andrea E. Bonny MD MD $^{1,3,4}$ ๑
}

${ }^{1}$ The Research Institute at Nationwide Children's Hospital, Columbus, Ohio

${ }^{2}$ Department of Psychiatry, University of Michigan, Ann Arbor, Michigan

${ }^{3}$ The Ohio State University, Columbus, Ohio

${ }^{4}$ Division of Adolescent Medicine, Nationwide Children's Hospital, Columbus, Ohio

\section{Correspondence}

Andrea E. Bonny, Nationwide Children's Hospital, 700 Children's Drive, Columbus, $\mathrm{OH}$ 43205, USA.

Email: andrea.bonny@nationwidechildrens.org

Funding information

Center for Clinical and Translational Science,

The Ohio State University, Grant/Award

Number: UL1TR001070; Clinical and

Translational Intramural Funding Program, The

Research Institute at Nationwide Children's

Hospital; The Ohio State University Center for

Integrative Health and Wellness

\begin{abstract}
Objective: To evaluate the effectiveness and tolerability of omega-3 polyunsaturated fatty acid (PUFA) supplementation for treatment of trait anxiety among adolescent females with restrictive anorexia nervosa (AN).

Method: A pilot double-blind, placebo-controlled randomized trial of adolescent females with AN ( $=24)$ entering Partial Hospitalization Program (PHP) from January 2015 to February 2016. Participants were randomized to four daily PUFA (2,120 mg eicosapentaenoic acid/600 $\mathrm{mg}$ docosohexaenoic acid) or placebo capsules for 12 weeks. A 9-item questionnaire of side effect frequency assessed medication tolerability. The Beck Anxiety Inventory-Trait measured anxiety at baseline, 6, and 12 weeks. Linear mixed models evaluated associations between randomization group and study outcomes. Twenty-two and 18 participants completed 6 and 12 weeks of data collection, respectively.

Results: Medication side effect scores were low and were not significantly different between randomization groups at Week $6(p=.20)$ or $12(p=.41)$. Mean trait anxiety score significantly $(p<.01)$ decreased from baseline to 12 weeks in both groups, and the rate of change over the course of time did not differ between omega-3 PUFA and placebo groups ( $p=.55$ ).

Conclusion: Omega-3 PUFA supplementation was well tolerated in adolescent females with AN. Although power to detect differences was limited, we found no evidence that omega-3 PUFA benefited anxiety beyond nutritional restoration.
\end{abstract}

\section{KEYWORDS}

adolescent, anorexia nervosa, anxiety, fatty acids, omega-3, randomized controlled trial

\section{1 | BACKGROUND}

Treatment of anorexia nervosa (AN) is complicated by high rates of co-morbid psychiatric diagnoses (Ulfvebrand, Birgegard, Norring, Hogdahl, \& von Hausswolff-Juhlin, 2015) and lack of effective pharmacologic interventions (Flament, Bissada, \& Spettigue, 2012). Anxiety disorders in particular are commonly comorbid with AN (Thornton, Dellava, Root, Lichtenstein, \& Bulik, 2011). Perfectionism, rigidity, compulsivity, and trait anxiety are elevated among individuals diagnosed with AN (Hildebrandt, Bacow, Markella, \& Loeb, 2012). Standard medication treatments for generalized anxiety, such as selective serotonin reuptake inhibitors (SSRIs), are generally ineffective in malnourished individuals with AN (Haleem, 2012).

There has been scientific interest in the utilization of omega-3 polyunsaturated fatty acids (PUFA) as treatment for several mental health disorders (Bozzatello, Brignolo, De Grandi, \& Bellino, 2016). Unlike SSRIs which require protein synthesis, omega-3 PUFA are 
hypothesized to alter brain phospholipid composition and enhance membrane fluidity, suggesting efficacy regardless of nutritional status (Carlezon Jr. et al., 2005).

Observational studies in adolescents with eating disorders have documented associations between depressive symptoms, low selfreported omega-3 PUFA consumption, and low omega-3 PUFA in erythrocyte membranes. In a population-based cohort study of female adolescents $(n=66)$, self-reported omega-3 and omega-6 fatty acid dietary intake were significantly inversely correlated with eating disorder and depressive symptoms among those with an eating disorder (Allen et al., 2013). In an earlier study exploring erythrocyte membrane fatty acid composition among 217 adolescents with eating disorders, lower proportions of omega-3 PUFA were similarly found to increase odds of depression (Swenne, Rosling, Tengblad, \& Vessby, 2011).

Supplementation trials have shown mixed results regarding omega-3 PUFA and anxiety. No omega-3 PUFA effects were observed in patients with obsessive compulsive disorder taking maximum doses of SSRIs (Fux, Benjamin, \& Nemets, 2004). Decreased anxiety was found in patients enrolled in a substance abuse treatment program supplemented with omega-3 PUFA (Buydens-Branchey, Branchey, \& Hibbeln, 2008). Decreased testrelated anxiety symptoms were observed in a non-clinical sample of medical students receiving omega-3 PUFA of similar composition to the current study (Kiecolt-Glaser, Belury, Andridge, Malarkey, \& Glaser, 2011). Mixed results of previous trials may be due to utilization of omega-3 PUFA of differing compositions in varying populations with different methodologies. Lack of standardization has limited our understanding of the relationship between omega-3 PUFA and anxiety.

With regards to AN and anxiety specifically, Barbarich et al. conducted a placebo-controlled randomized trial of nutritional supplements, containing omega-3 PUFA composed of $600 \mathrm{mg}$ docosohexaenoic acid (DHA) and $180 \mathrm{mg}$ of arachadonic acid daily, in young adults (mean age $23.0 \pm 6.3$ years) with AN receiving fluoxetine. No significant differences in change in anxiety were evident between those on and off supplements, but the study was underpowered to examine these effects (Barbarich et al., 2004). To our knowledge, there have been no systematic trials of omega-3 PUFA in adolescents with AN. The objective of this pilot randomized, placebocontrolled study was to evaluate the effectiveness and tolerability of omega-3 PUFA supplementation for improvement in trait anxiety in female adolescents with restrictive AN.

\section{2 | METHODS}

This double-blind, placebo-controlled, single center pilot study was approved by Nationwide Children's Hospital's ( $\mathrm{NCH}$ ) Institutional Review Board and the U.S. Food and Drug Administration (IND 117431). It was registered at clinicaltrials.gov (NCT01933243). The study population consisted of adolescent females aged 12-21 admitted into the $\mathrm{NCH}$ Eating Disorders Partial Hospitalization Program (PHP) for treatment of AN, restrictive subtype, from January 2015 to February 2016. Diagnosis was made via clinical interviews and consensus of the multidisciplinary team based on the Diagnostic and Statistical Manual of Mental Disorders Fifth Edition (American Psychiatric Association, 2013). Every patient was first assessed by an Adolescent Medicine physician, a licensed dietitian, and a trained eating disorder therapist. This assessment established the preliminary diagnosis, which was then confirmed by the program's psychiatrist during intake visit, occurring $\sim 48 \mathrm{hr}$ prior to the start of PHP.

Study exclusion criteria included: (1) inability to take pills, (2) comorbid medical condition affecting appetite or weight (e.g., inflammatory bowel disease), (3) co-morbid psychiatric diagnoses affecting appetite and weight (e.g., bipolar disorder), (4) currently taking omega-3 PUFA supplements, (5) unable to participate in the study for 12 consecutive weeks. SSRI use was not an exclusionary criterion for enrollment; 21 of the 24 participants were on an SSRI during the study.

Of 41 potential participants assessed prior to expiration of funding, 2 did not meet inclusion criteria, 15 declined to participate, and 24 enrolled and were randomized in a 1:1 ratio to either omega-3 PUFA supplements (Nordic Naturals ${ }^{\circledR}$ ProEPA ${ }^{\mathrm{TM}}$ Xtra, Watsonville, CA) or placebo (Nordic Naturals ${ }^{\circledR}$, Watsonville, CA), four capsules orally daily for 12 weeks (Figure 1). A block randomization scheme was electronically generated by $\mathrm{NCH}$ Investigational Drug Service Pharmacy (IDS) to randomize participants into blocks of 8 (Suresh, 2011). After consent, study staff contacted IDS who then assigned participants to a treatment arm sequentially from a prepared list. Study staff were not involved with randomization and were unaware of upcoming allocation. Participants, study staff, and statistician were blinded to drug assignment.

The four omega-3 PUFA capsules provided a total daily dose of 2,120 mg eicosapentaenoic acid (EPA), $600 \mathrm{mg}$ DHA, and $404 \mathrm{mg}$ of other omega-3 PUFA. This high EPA product was chosen based on prior data supporting effectiveness for anxiety (Kiecolt-Glaser et al., 2011). Supplements contained lemon essential oil to mask potential fishy aftertaste. Placebo capsules were identical color, size, and flavor but contained predominantly soybean oil (3,960 mg total daily dose) and negligible omega-3 PUFA (40 mg total daily dose).

Study visits occurred at baseline, 6 , and 12 weeks. Of 24 enrolled participants, 22 completed 6 weeks of data collection, and 18 completed 12 weeks (Figure 1). Reasons for study non-completion included: loss to follow-up $(n=4)$ and self-withdrew $(n=2)$.

Measures conducted at each study visit included the Beck Anxiety Inventory-Trait (BAIT) (Kohn, Kantor, DeCicco, \& Beck, 2008), Center for Epidemiologic Studies Depression Scale (CES-D) (Radloff, 1991), and Eating Attitudes Test (EAT-26) (Garner, Olmsted, Bohr, \& Garfinkel, 1982). Data were captured electronically and stored within REDCap ${ }^{\mathrm{TM}}$. Surveys were programmed to require a response for all fields.

The BAIT is a 21-item self-report measure of trait anxiety severity rated on a 4-point Likert scale ( 0 = rarely or never; 3 = almost always). It has shown acceptable reliability and validity in an adolescent psychiatric inpatient population (Osman et al., 2002). BAIT scores over 26 indicate severe anxiety, scores 16-25 moderate anxiety, scores 8-15 mild anxiety, and scores 0-7 minimal anxiety.

The CES-D is a well-validated instrument for self-report of depression symptoms (Radloff, 1991). The 20-item survey uses a 


\section{Enrollment}

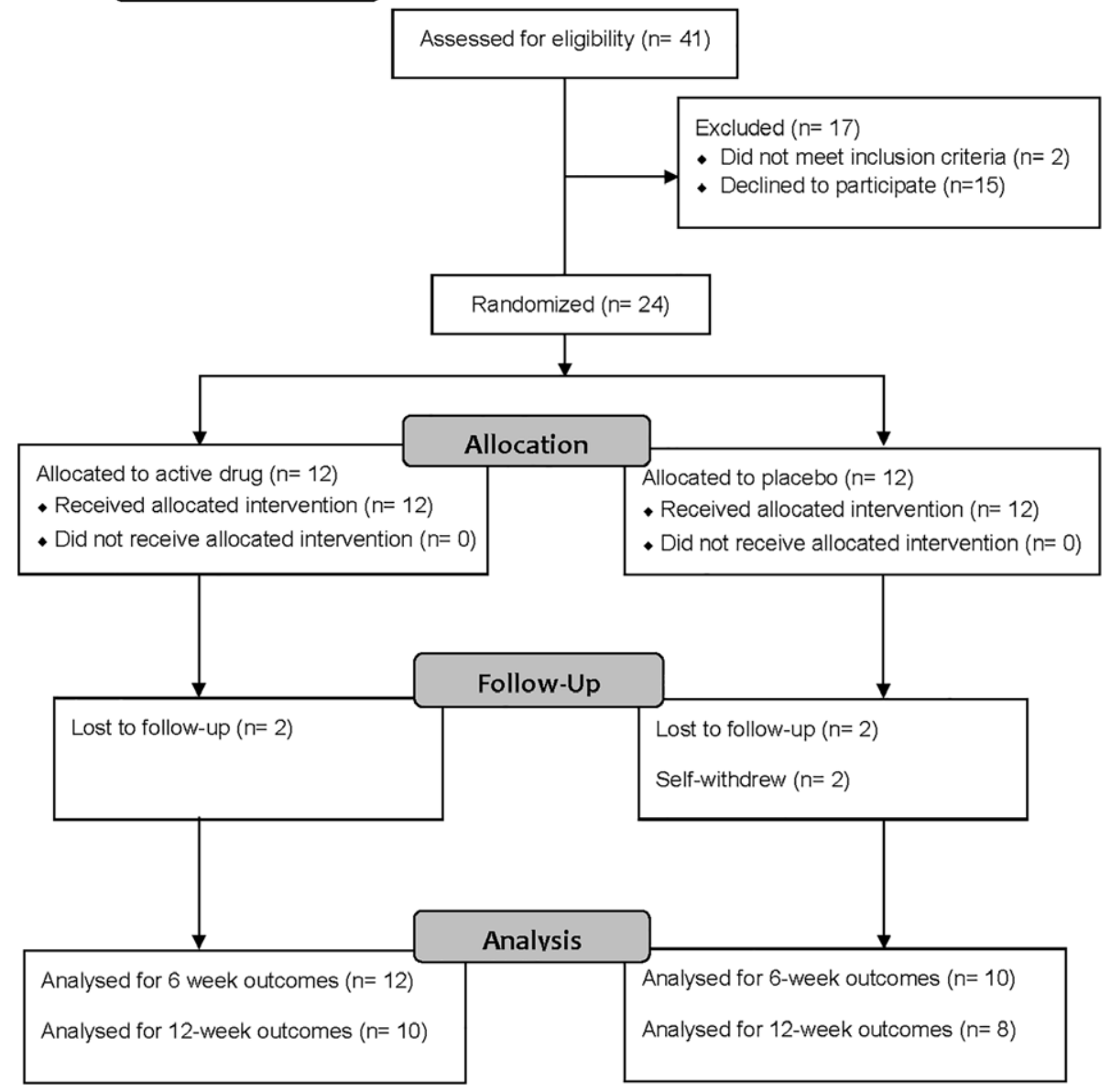

FIGURE 1 Participant flow diagram

4-point Likert scale indicating frequency of agreement with statements $(0=$ rarely; $3=$ most of the time) with a range of $0-60$. A score of 24 or higher in females is indicative of high depressive symptoms.

Eating Attitudes Test (EAT-26) is a standardized measure of prevalent symptoms and concerns characteristic of eating disorders (Garner et al., 1982). The scale consists of 26 items rated on a 6-point scale. Subscales include dieting; bulimia and food preoccupation; and oral control. Although the EAT-26 is not a diagnostic tool, scores $\geq 20$ are indicative of a heightened risk of eating disorder diagnosis.

At follow-up visits, medication tolerability was assessed via selfreport of nine potential side effects (e.g., diarrhea, burping), scored on a 5 -point frequency scale $(0=$ never; $4=$ very frequently). Total side effect score ranged from 0 to 36 . Higher scores indicated lower medication tolerability.

Chart review was conducted to obtain height and weight closest in date to research visits. Height was assessed using a wall-mounted stadiometer with participant in stocking feet; weight was measured with a SECA digital scale with participant gowned and in stocking feet. Height and weight were not assessed as part of the research protocol as standard of care in the Eating Disorders Program includes regular measurement of both.

\section{1 | Statistical analyses}

All randomized participants were included in the analyses, in their initially randomized group, regardless of protocol deviation or adherence (McCoy, 2017). The primary outcome was change in trait anxiety (BAIT score) over the course of time. Power analysis prior to study initiation determined that we would need 20 per group to have $75 \%$ power to detect a presumed mean difference of 5 between groups with a standard deviation of 6. Secondary outcomes included BMI, medication tolerability, EAT-26, and CES-D scores.

Patient demographic and clinical characteristics were reported as means (standard deviations) for continuous variables and frequencies (percentages) for categorical variables. Differences in baseline characteristics were compared between study groups using Student's $t$ and chi-square tests.

Linear mixed models with random intercepts to account for repeated measures were used to evaluate the primary and secondary outcomes over time. Models included main effects for study group and time and a group by time interaction term to test for group differences in rate of change over time. Model-based estimates were reported as least square means ( $95 \%$ confidence intervals). Hypothesis testing was conducted at an alpha of $.05 ; p$-values $<.05$ were 
considered statistically significant. Analyses were conducted using SAS version 9.4 (SAS Institute, Cary, NC).

\section{3 | RESULTS}

At baseline, the study groups (omega-3 PUFA vs. placebo) did not differ in age, race, height, weight, BMI, EAT-26, or trait anxiety score (Table 1). Amenorrhea was present in over $50 \%$ of participants.

Overall, side effect scores were low, indicating good medication tolerability (Table 2). Mean side effect scores were not significantly different between randomization groups at Week $6(p=.20)$ or $12(p=.41)$. Mean side effect scores significantly decreased from Week 6 to Week 12 for the omega-3 PUFA group ( $p=.02$ ) but not the placebo group ( $p=.15$ ); however, rate of change over time did not differ between groups $(p=.54)$. No serious drug-related adverse events occurred in either group.

There were no significant differences between groups at any time point in measures of weight, eating disorder symptoms, or depression (Table 2). For both groups, improvement in these measures was evident over 12 weeks, that is, there was a significant main effect of time for BMI, EAT-26, and depression in the expected directions.

Mean trait anxiety score was higher in the omega-3 PUFA group at each time point, with the difference between groups being significant at 6 and 12 weeks (Table 2). Scores in the omega-3 PUFA group indicated severe anxiety at baseline and mild anxiety at 12 weeks; scores in the placebo group indicated moderate anxiety at baseline and minimal anxiety at 12 weeks. Mean trait anxiety significantly $(p<.01)$ decreased from baseline to 12 weeks in both groups, and the rate of change in trait anxiety over the course of the study did not differ between the two study groups ( $p=.55$ ).

\section{4 | DISCUSSION}

In this pilot randomized controlled trial, female adolescents in PHP for restrictive AN found omega-3 supplementation tolerable. Reported side effects were infrequent, decreased over time, and were similar for omega-3 PUFA and placebo. Overall, no significant differences were found in change in trait anxiety, depression, eating disorder symptoms, and BMI over 12 weeks regardless of randomization group. Our findings suggest that omega-3 PUFA supplementation does not confer additional benefit beyond nutritional restoration for adolescents initiating PHP.

Study power was limited by small sample size and mean difference between groups which was smaller than anticipated. Despite the null finding, the high tolerability of omega-3 PUFA supplementation may warrant further study, particularly in the outpatient treatment setting. It is possible that potential beneficial effects of omega-3 PUFA were modest and lost in the known significant effects of intensive nutritional rehabilitation experienced in PHP.

Other limitations include reliance on self-reported medication adherence, no measurement of blinding success, and no follow-up on participants after study completion. Given that our sample was entirely female and that the threshold for PHP admission likely varies by institution, our findings may not be generalizable to other populations.

In conclusion, omega-3 PUFA supplementation was well tolerated in a population of adolescent females with moderate to severe AN. We found no evidence that omega-3 PUFA supplementation

TABLE 1 Baseline participant characteristics by study group

\begin{tabular}{|c|c|c|c|}
\hline Characteristic & Omega-3 PUFAa $(n=12)$ & Placebo $(n=12)$ & $p$ value \\
\hline Age (years), mean (SD) & $15.0(1.3)$ & $14.4(1.8)$ & .34 \\
\hline Race, $n(\%)$ & & & .24 \\
\hline White non-Hispanic & $12(100.0)$ & $10(83.3)$ & \\
\hline Black non-Hispanic & $0(0.0)$ & $2(16.7)$ & \\
\hline Height (cm), mean(SD) & $164.3(8.0)$ & $162.1(9.0)$ & .54 \\
\hline Weight (kg), mean (SD) & $52.8(7.6)$ & $49.5(9.2)$ & .35 \\
\hline Body mass index $\left(\mathrm{kg} / \mathrm{m}^{2}\right)$, mean $(S D)$ & $19.6(2.0)$ & $18.8(2.1)$ & .33 \\
\hline Reported maximum weight (Ibs), mean (SD) & $125.2(11.6)$ & $124.1(36.6)$ & .93 \\
\hline Reported minimum weight (lbs), mean (SD) & $101.7(13.7)$ & $96.8(22.8)$ & .53 \\
\hline Recent weight loss (Ibs/month), mean (SD) & $4.0(2.1)$ & $6.1(3.4)$ & .08 \\
\hline Presence of amenorrhea, $n$ (\%) & $7(58.3)$ & $6(50.0)$ & .58 \\
\hline \multicolumn{4}{|l|}{ Eating disorder symptoms score, ${ }^{\mathrm{b}}$ mean $(S D)$} \\
\hline Dieting & $21.8(11.4)$ & $23.2(12.0)$ & .78 \\
\hline Bulimia & $5.6(3.9)$ & $6.9(4.3)$ & .44 \\
\hline Oral control & $10.3(5.2)$ & $11.3(5.5)$ & .65 \\
\hline Total & 37.7 (19.2) & $41.3(20.7)$ & .66 \\
\hline Trait anxiety, ${ }^{\mathrm{c}}$ mean (SD) & $26.4(10.0)$ & $18.9(10.3)$ & .08 \\
\hline
\end{tabular}

a Omega-3 polyunsaturated fatty acid supplementation with 2,120 mg eicosapentaenoic acid +600 mg docosohexaenoic acid daily.

b Measured by The Eating Attitudes Test (EAT-26).

c Measured by The Beck Anxiety Inventory-Trait (BAIT). 
TABLE 2 Linear mixed model estimates of medication side effect, weight, eating disorder, depression, and anxiety measures over time by study group

\begin{tabular}{|c|c|c|c|c|}
\hline \multicolumn{4}{|c|}{ Omega-3 PUFA ${ }^{a}$} & \multirow[t]{2}{*}{ Placebo } \\
\hline \multicolumn{4}{|l|}{ Sample size $(n)$} & \\
\hline Baseline & & & & 12 \\
\hline 6 weeks & & & & 10 \\
\hline 12 weeks & & & & 8 \\
\hline Measure & $\begin{array}{l}\text { Omega-3 PUFA } \\
\text { Least squares mean }(95 \% \mathrm{CI})\end{array}$ & $\begin{array}{l}\text { Placebo } \\
\text { Least squares mean }(95 \% \mathrm{Cl})\end{array}$ & $\begin{array}{l}\text { Cohen's D } \\
\text { Effect size }(95 \% \mathrm{Cl})\end{array}$ & $p$ value \\
\hline Medication side effects score ${ }^{b}$ & & & & $.54^{\mathrm{c}}$ \\
\hline Baseline & $\mathrm{n} / \mathrm{a}$ & $\mathrm{n} / \mathrm{a}$ & $\mathrm{n} / \mathrm{a}$ & $\mathrm{n} / \mathrm{a}$ \\
\hline 6 weeks & $9.7(6.1,13.2)$ & $6.2(2.1,10.3)$ & $0.52(-0.4,1.4)^{d}$ & $.20^{\mathrm{d}}$ \\
\hline 12 weeks & $6.4(2.7,10.0)$ & $4.1(-0.1,8.3)$ & $0.50(-0.4,1.5)^{d}$ & $.41^{d}$ \\
\hline Body mass index $\left(\mathrm{kg} / \mathrm{m}^{2}\right)$ & & & & $.42^{c}$ \\
\hline Baseline & $19.6(18.3,20.8)$ & $18.8(17.5,20.0)$ & $0.41(-0.4,1.2)^{d}$ & $.34^{d}$ \\
\hline 6 weeks & $21.6(20.3,22.8)$ & $20.4(19.1,21.7)$ & $0.37(-0.5,1.2)^{d}$ & $.19^{\mathrm{d}}$ \\
\hline 12 weeks & $21.9(20.7,23.2)$ & $20.6(19.3,21.8)$ & $0.25(-0.7,1.2)^{d}$ & $.13^{d}$ \\
\hline Total EAT score & & & & $.21^{\mathrm{c}}$ \\
\hline Baseline & $37.7(26.2,49.2)$ & $41.3(29.8,52.8)$ & $-0.18(-1.0,0.6)^{d}$ & $.65^{d}$ \\
\hline 6 weeks & $26.9(15.4,38.4)$ & $28.3(16.2,40.4)$ & $-0.07(-0.9,0.8)^{d}$ & $.87^{d}$ \\
\hline 12 weeks & $23.2(11.2,35.2)$ & $14.0(1.2,26.8)$ & $0.51(-0.4,1.5)^{d}$ & $.30^{d}$ \\
\hline Depression score ${ }^{f}$ & & & & $.94^{\mathrm{c}}$ \\
\hline Baseline & $28.8(24.3,33.4)$ & $25.1(20.5,29.6)$ & $0.44(-0.4,1.3)^{d}$ & $.25^{\mathrm{d}}$ \\
\hline 6 weeks & $26.1(21.5,30.6)$ & $22.0(17.2,26.9)$ & $0.45(-0.4,1.3)^{d}$ & $.23^{d}$ \\
\hline 12 weeks & $21.0(16.2,25.8)$ & $16.2(11.1,21.3)$ & $0.42(-0.5,1.4)^{d}$ & $.18^{d}$ \\
\hline Trait anxiety score ${ }^{\mathrm{g}}$ & & & & $.55^{c}$ \\
\hline Baseline & $26.4(20.7,32.1)$ & $18.9(13.2,24.6)$ & $0.74(-0.1,1.6)^{d}$ & $.07^{d}$ \\
\hline 6 weeks & $22.2(16.5,27.9)$ & $11.0(5.1,17.0)$ & $1.11(0.2,2.0)^{d}$ & $.01^{d}$ \\
\hline 12 weeks & $15.4(9.4,21.3)$ & $6.3(0.0,12.6)$ & $0.99(0.0,2.0)^{d}$ & $.04^{d}$ \\
\hline
\end{tabular}

a Omega-3 polyunsaturated fatty acid supplementation with 2,120 mg eicosapentaenoic acid +600 mg docosohexaenoic acid daily.

b Measured by a 9-item questionnaire (score range 0-36) addressing frequency of potential side effects.

c Value for difference in rate of change (interaction effect between group and time).

${ }^{\mathrm{d}}$ Value for between group difference.

e Measured by The Eating Attitudes Test (EAT-26).

${ }^{f}$ Measured by the Center for Epidemiologic Studies Depression Scale (CES-D).

$g$ Measured by the Beck Anxiety Inventory-Trait (BAIT).

provides additional benefit beyond nutritional restoration for patients enrolled in PHP. Future study with larger sample sizes should focus on patients in less intensive treatment programs, incorporate physiological measures of compliance, and explore PUFA supplements of varying composition for potential effectiveness.

\section{ACKNOWLEDGMENTS}

This study was supported by the Clinical and Translational Intramural Funding Program at the Research Institute at Nationwide Children's Hospital and the Center for Integrative Health and Wellness at The Ohio State University. Additional support received from OSU CTSA, Grant no. UL1TR001070. Drug (active and placebo) provided in kind by Nordic Naturals ${ }^{\circledR}$.

\section{ORCID}

\section{REFERENCES}

Allen, K. L., Mori, T. A., Beilin, L., Byrne, S. M., Hickling, S., \& Oddy, W. H. (2013). Dietary intake in population-based adolescents: Support for a relationship between eating disorder symptoms, low fatty acid intake and depressive symptoms. Journal of Human Nutrition and Dietetics, 26(5), 459-469. https://doi.org/10.1111/jhn.12024

American Psychiatric Association (Ed.). (2013). Diagnostic and statistical manual of mental disorders (5th ed.). Arlington, VA: American Psychiatric Publishing, Inc.

Barbarich, N. C., McConaha, C. W., Halmi, K. A., Gendall, K., Sunday, S. R., Gaskill, J., ... Kaye, W. H. (2004). Use of nutritional supplements to increase the efficacy of fluoxetine in the treatment of anorexia nervosa. International Journal of Eating Disorders, 35(1), 10-15. https://doi. org/10.1002/eat.10235

Bozzatello, P., Brignolo, E., De Grandi, E., \& Bellino, S. (2016). Supplementation with omega-3 fatty acids in psychiatric disorders: A review of literature data. Journal of Clinical Medicine, 5(8), E67. https://doi.org/10. 3390/jcm5080067.

Buydens-Branchey, L., Branchey, M., \& Hibbeln, J. R. (2008). Associations between increases in plasma $n-3$ polyunsaturated fatty acids following supplementation and decreases in anger and anxiety in substance abusers. Progress in Neuro-Psychopharmacology and Biological Psychiatry, 32(2), 568-575. https://doi.org/10.1016/j.pnpbp.2007.10.020 
Carlezon, W. A., Jr., Mague, S. D., Parow, A. M., Stoll, A. L., Cohen, B. M., \& Renshaw, P. F. (2005). Antidepressant-like effects of uridine and omega-3 fatty acids are potentiated by combined treatment in rats. Biological Psychiatry, 57(4), 343-350. https://doi.org/10.1016/j. biopsych.2004.11.038

Flament, M. F., Bissada, H., \& Spettigue, W. (2012). Evidence-based pharmacotherapy of eating disorders. International Journal of Neuropsychopharmacology, 15(2), 189-207. https://doi.org/10.1017/S1461145711000381

Fux, M., Benjamin, J., \& Nemets, B. (2004). A placebo-controlled cross-over trial of adjunctive EPA in OCD. Journal of Psychiatric Research, 38(3), 323-325. https://doi.org/10.1016/S0022-3956(03) 00077-3

Garner, D. M., Olmsted, M. P., Bohr, Y., \& Garfinkel, P. E. (1982). The eating attitudes test: Psychometric features and clinical correlates. Psychological Medicine, 12(4), 871-878.

Haleem, D. J. (2012). Serotonin neurotransmission in anorexia nervosa. Behavioural Pharmacology, 23(5-6), 478-495. https://doi.org/10. 1097/FBP.Ob013e328357440d

Hildebrandt, T., Bacow, T., Markella, M., \& Loeb, K. L. (2012). Anxiety in anorexia nervosa and its management using family-based treatment. European Eating Disorders Review, 20(1), e1-e16. https://doi.org/10. 1002/erv.1071

Kiecolt-Glaser, J. K., Belury, M. A., Andridge, R., Malarkey, W. B., \& Glaser, R. (2011). Omega-3 supplementation lowers inflammation and anxiety in medical students: A randomized controlled trial. Brain, Behavior and Immunity, 25(8), 1725-1734. https://doi.org/10.1016/j. bbi.2011.07.229

Kohn, P. M., Kantor, L., DeCicco, T. L., \& Beck, A. T. (2008). The Beck anxiety inventory-trait (BAIT): A measure of dispositional anxiety not contaminated by dispositional depression. Journal of Personality Assessment, 90(5), 499-506. https://doi.org/10.1080/00223890802248844

McCoy, C. E. (2017). Understanding the intention-to-treat principle in randomized controlled trials. Western Journal of Emergency Medicine, 18(6), 1075-1078. https://doi.org/10.5811/westjem.2017.8.35985

Osman, A., Hoffman, J., Barrios, F. X., Kopper, B. A., Breitenstein, J. L., \& Hahn, S. K. (2002). Factor structure, reliability, and validity of the Beck anxiety inventory in adolescent psychiatric inpatients. Journal of Clinical Psychology, 58(4), 443-456.

Radloff, L. S. (1991). The use of the Center for Epidemiologic Studies Depression Scale in adolescents and young adults. Journal of Youth and Adolescence, 20(2), 149-166. https://doi.org/10.1007/BF01537606

Suresh, K. (2011). An overview of randomization techniques: An unbiased assessment of outcome in clinical research. Journal of Human Reproductive Sciences, 4(1), 8-11. https://doi.org/10.4103/0974-1208.82352

Swenne, I., Rosling, A., Tengblad, S., \& Vessby, B. (2011). Omega-3 polyunsaturated essential fatty acids are associated with depression in adolescents with eating disorders and weight loss. Acta Paediatrica, 100(12), 1610-1615. https://doi.org/10.1111/j.1651-2227.2011.02400.x

Thornton, L. M., Dellava, J. E., Root, T. L., Lichtenstein, P., \& Bulik, C. M. (2011). Anorexia nervosa and generalized anxiety disorder: Further explorations of the relation between anxiety and body mass index. Journal of Anxiety Disorders, 25(5), 727-730. https://doi.org/10.1016/j. janxdis.2011.03.010

Ulfvebrand, S., Birgegard, A., Norring, C., Hogdahl, L., \& von Hausswolff-Juhlin, Y. (2015). Psychiatric comorbidity in women and men with eating disorders results from a large clinical database. Psychiatry Research, 230(2), 294-299. https://doi.org/10.1016/j.psychres.2015.09.008

\section{SUPPORTING INFORMATION}

Additional supporting information may be found online in the Supporting Information section at the end of the article.

How to cite this article: Manos BE, Bravender TD, Harrison TM, et al. A pilot randomized controlled trial of omega-3 fatty acid supplementation for the treatment of anxiety in adolescents with anorexia nervosa. Int J Eat Disord. 2018;51:1367-1372. https://doi.org/10.1002/eat.22964 